\title{
PASCHEN-BACK EFFECT OF THE LITHIUM RESONANCE DOUBLET IN SUNSPOTS
}

\author{
P. MALTBY \\ Institute of Theoretical Astrophysics, University of Oslo, Norway
}

\begin{abstract}
The formation of the resonance doublet lines of $\mathrm{Li}^{6}$ and $\mathrm{Li}^{7}$ in sunspots is discussed. It is shown that the magnetic splitting of the lines must be determined according to the (partial) PaschenBack pattern. As a first approximation to the problem a detailed calculation of the line profile is given for the case of pure absorption and local thermodynamic equilibrium.
\end{abstract}

\section{Introduction}

The $\mathrm{Li}$ I resonance lines at $\lambda 6708$ have been considered by several authors, in particular in connection with abundance determination.

In the case of penumbral/umbral spectrum the resonance lines have been treated as magnetically inactive (e.g. Greenstein and Richardson, 1951) or as affected by the Zeeman splitting (e.g. Wiehr et al., 1968). However, the separation of the ${ }^{2} P_{3 / 2}$ and ${ }^{2} P_{1 / 2}$ levels of $\mathrm{LiI}$ is so small $(0.151 \AA)$ that the magnetic splitting in sunspot fields is comparable to the doublet separation. Thus, the intensity and wavelength position of the line components are determined according to the Paschen-Back pattern. Recently, Traub (1968) has taken the Paschen-Back effect into account - he has also considered the effect of the nuclear spin, but found that this additional effect may be neglected for magnetic field strengths above approximately $1000 \mathrm{G}$.

Let us consider the problem of the formation of the $\mathrm{Li}$ I $\lambda 6708$ line, consisting of the $\mathrm{Li}^{7}$ doublet $\lambda \lambda 6707.761,6707.912$ and the $\mathrm{Li}^{6}$ doublet $\lambda \lambda 6707.921,6708.072$. Each doublet is split into $6+4=10$ components in the magnetic field (neglecting 2 components associated with the nuclear spin). Thus, the first problem is to determine the intensity and wavelength position of each component. This information may be taken from Traub (1968) or several earlier authors. The second problem is the formation of the magnetically active lines in the spot atmosphere. Traub (1968) tried to solve this problem by establishing a linear approximation to the Planck function at each depth and assuming that Seares' formulae could be applied. We will follow another approach by using the line formation theory of Kjeldseth Moe (1968) and apply this theory to lines blended by other magnetically active lines.

As we are dealing with a resonance doublet, deviations from local thermodynamic equilibrium (LTE) as well as line scattering may be important. The calculations are based on the assumptions of pure absorption and LTE and should accordingly be regarded as a first, rough approximation to this line formation problem.

\section{Theoretical Considerations and Results}

The wave mechanical treatment of magnetic splitting at intermediate strengths of 
magnetic field was given by Darwin (1927). Numerical examples are given by Darwin (1928) for $s-p$ and $p$ - $d$ doublets and the $s-p$ triplet. In our case, the $s-p$ doublet, the problem was considered already by Voigt (1913) using a classical approach. Sommerfeld (1922) gave the quantum theory modification of Voigt's work and Heisenberg and Jordan (1926) gave a quantum mechanical treatment for doublets. For our numerical work we will use the results given by Darwin (1928).

Let us consider the vector model for $\mathrm{Li}$, in which only the outermost electron plays a role. As a result of the spin moment, $\mathbf{s}$, the orbital plane precesses about the total angular momentum, $\mathbf{j}$. We here regard $\mathbf{j}$ as composed of the orbital moment $\mathbf{l}$ and the spin moment, $\mathbf{s}$. We may regard the precession frequency of $\mathbf{s}$ and $\mathbf{l}$ about $\mathbf{j}$ as a measure of the doublet separation (e.g. Pauling and Goudsmit, 1930). In dealing with the Zeeman effect it is often useful to regard the splitting of a level as a measure of the precession frequency of $\mathbf{j}$ about the external magnetic field, $\mathbf{H}$. For the Zeeman effect we may assume that the precession of $\mathbf{s}$ and $\mathbf{l}$ about $\mathbf{j}$ is much faster than the Larmor precession. For strong magnetic fields and small doublet separation the precession frequencies become comparable and another approach must be used.

For a very strong magnetic field $\mathbf{l}$ and $\mathbf{s}$ wil lboth precess independently about the external field direction. For the Li doublet in sunspot spectra we are interested in the case where the external magnetic field is of the same order of magnitude as the internal magnetic field. In this case the precessions of $\mathbf{s}$ and $\mathbf{l}$ about $\mathbf{j}$ are not uniform, whereas the total projection of the mechanical moment on $\mathbf{H}$ is independent of magnetic field strength. The quantum mechanical calculations are done by taking into account simultaneously the magnetic field and the spin-orbit interaction. The perturbation theory used in quantum mechanics will not be given here. For a detailed discussion of the (partial) Paschen-Back effect we refer to Bethe and Salpeter (1957) and references given there.

Let us next consider the line formation of magnetically active lines. If we assume that the magnetic field is homogeneous and that the line is formed by pure absorption we may apply the solution given by Kjeldseth Moe (1968). A formal solution may also be

TABLE I

Data used in the numerical calculations

\begin{tabular}{ll} 
Parameter & Value/Source \\
\hline Magnetic pattern & Darwin (1928) \\
$f$-value & Bickel et al. (1969) \\
Sunspot model & $\Delta \theta=0.48$ model of Zwaan (1965) \\
Continuum absorption coefficient & Bode $(1965)$ \\
Abundance of $\mathrm{Li}$ & $\log N(\mathrm{Li})=0.80$, Engvold et al. (1970) \\
Isotope ratio $\mathrm{Li}^{8} / \mathrm{Li}^{7}$ & $0.05($ assumed value) \\
Position on solar disc & Centre \\
Partition function & Bascheck et al. (1966) \\
Van der Waal damping & $a=0.18$, see Aller (1963) \\
Turbulent velocity & zero (assumed value)
\end{tabular}


found in the case that scattering is important. However, the scattering matrix for the line must be derived and this is generally relatively difficult unless we restrict the discussion to a particular model atmosphere (as a Milne-Eddington atmosphere). Thus, as a first approximation we will regard the lithium line as formed by pure absorption. We will further assume that the sunspot atmosphere is in LTE and that the Doppler width of the line absorption coefficient may be regarded as constant through-

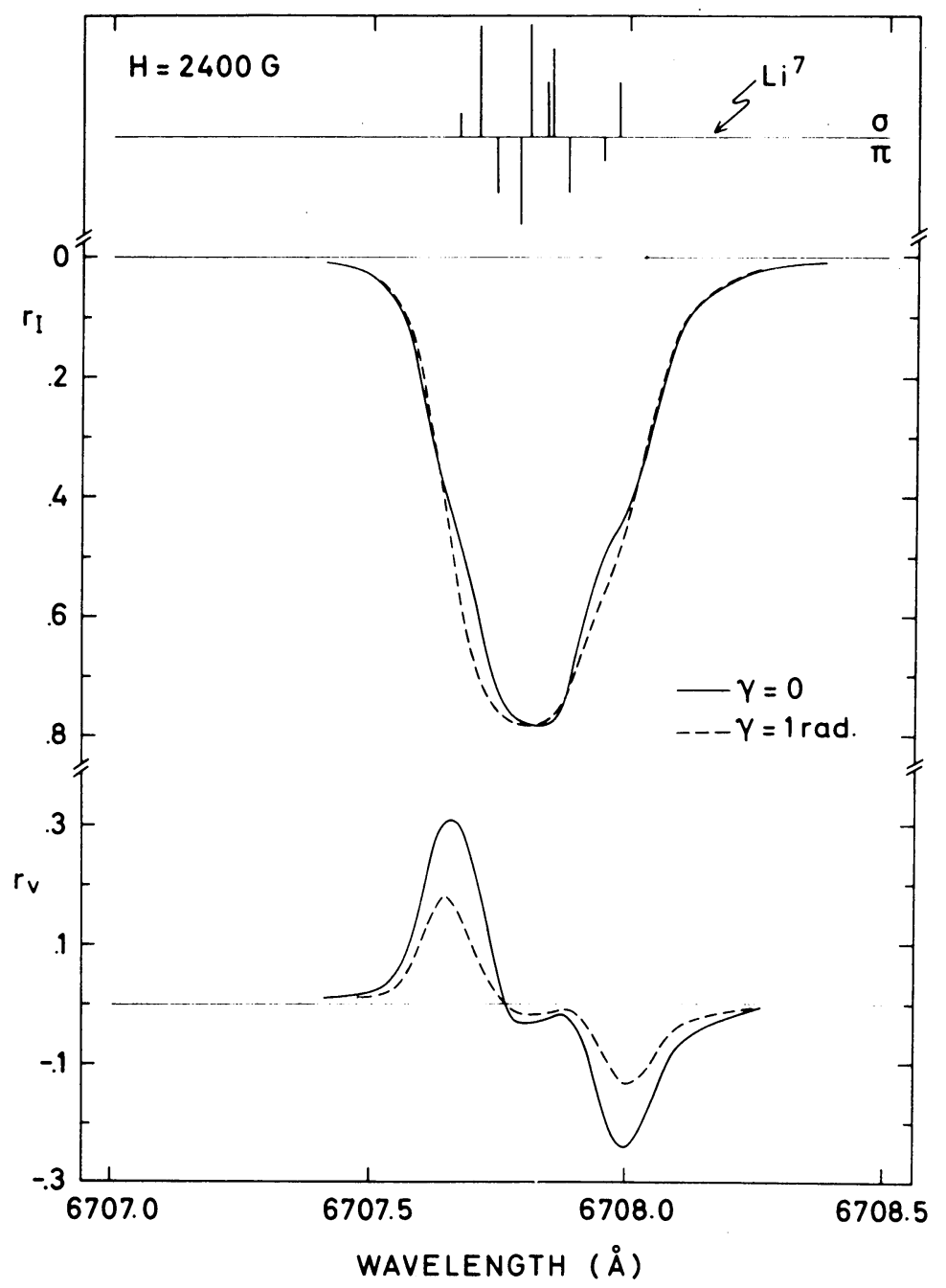

Fig. 1.

Figs. 1-4. The line depth $r_{I}=\left(I_{0}-I\right) / I_{0}$ and the fraction of circular polarization $r_{v}=-V / I_{0}(V$ is the Stokes parameter) as function of wavelength for the $\mathrm{Li}^{6}$ and $\mathrm{Li}^{7}$ resonance doublets. The PaschenBack pattern is indicated (top) for the $\mathrm{Li}^{7}$ components - the pattern is identical for $\mathrm{Li}^{6}$. The magnetic field strength, $H$, is given in the figure. The calculations are done for an angle $\gamma=0$ and 1 radian between the magnetic field direction and the line of sight. 
out the atmosphere. With these assumptions the same type of polarization will be absorbed and emitted at all depths at a fixed frequency within the line. The solution given by Kjeldseth Moe (1968) is obtained by regarding the light as composed of two independent light beams of elliptically polarized light in opposite states of polarization. In this way the problem may be transformed to a line formation problem similar to the non-magnetic case.

When two or more magnetically active lines blend with each other different types of polarization will generally be absorbed at different depths in the atmosphere. In the general case the type of solution outlined above is therefore not applicable, as the contribution functions for the line profiles are different. However, in the case of the lithium resonance doublet both lines start from the same atomic level and the contribution

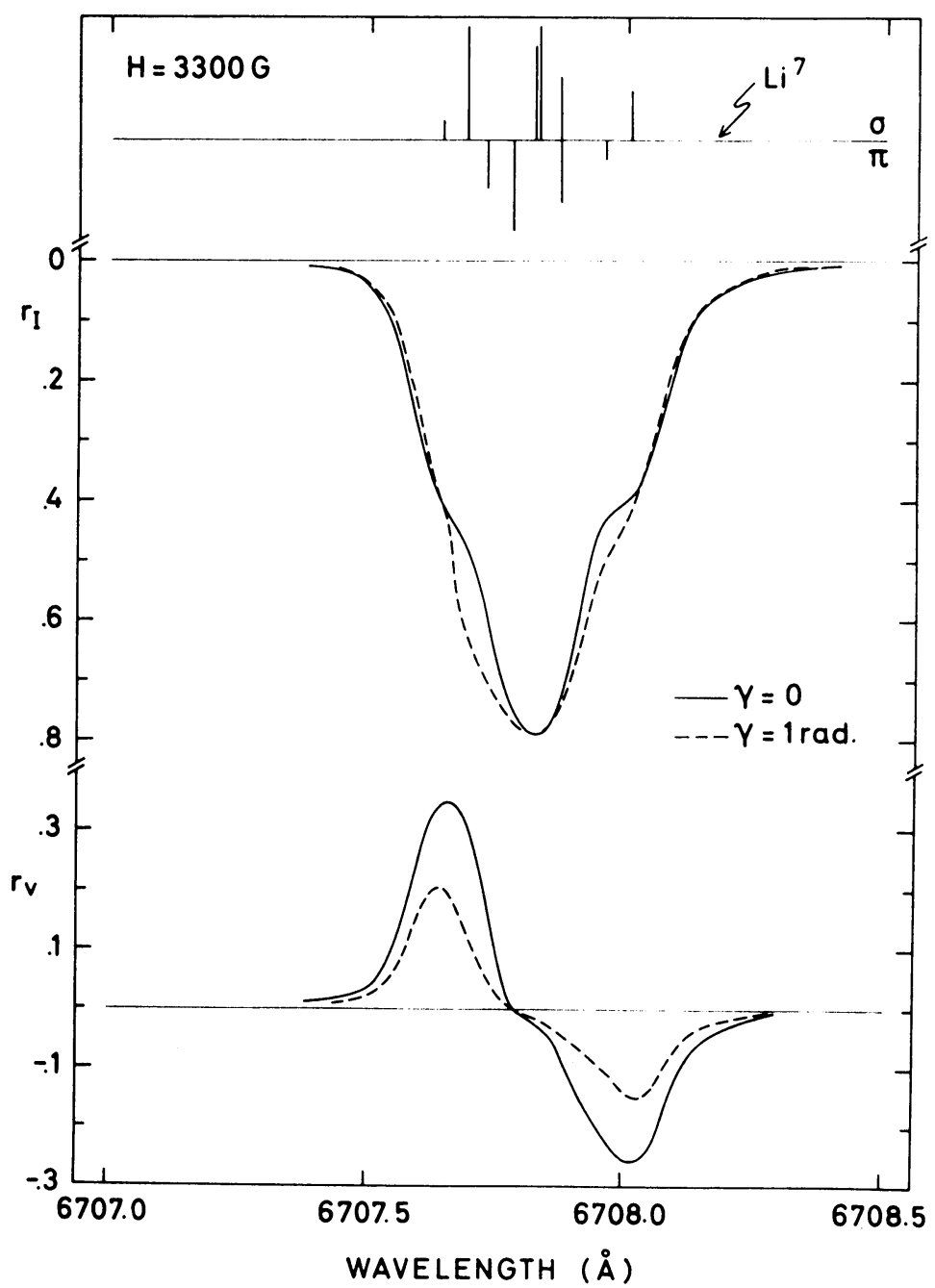

Fig. 2. 
functions are identical except for a numerical factor. This numerical factor is determined by the oscillator strength and the $\mathrm{Li}^{6} / \mathrm{Li}^{7}$ isotope ratio. Accordingly, we have calculated the composite line profile for the two doublets using the input data given in Table I. A more detailed account of the line calculation is given by Engvold et al. (1970).

The results of the calculations are shown in Figures 1-4 for different values of the magnetic field strength. Two values of the angle $\gamma$ between the line of sight and the magnetic field have been used in the calculations. Figures 1-4 give the line depth $r_{I}$ for the total intensity as well as the quantity $r_{v}$ for the circularly polarized intensity. These quantities are defined as $r_{I}=\left(I_{0}-I\right) / I_{0}$ and $r_{v}=-V / I_{0}$, where $I$ and $I_{0}$ are the intensities in the line and in the continuum, respectively and $V$ is the Stokes parameter that determines the fraction of circularly polarized light.

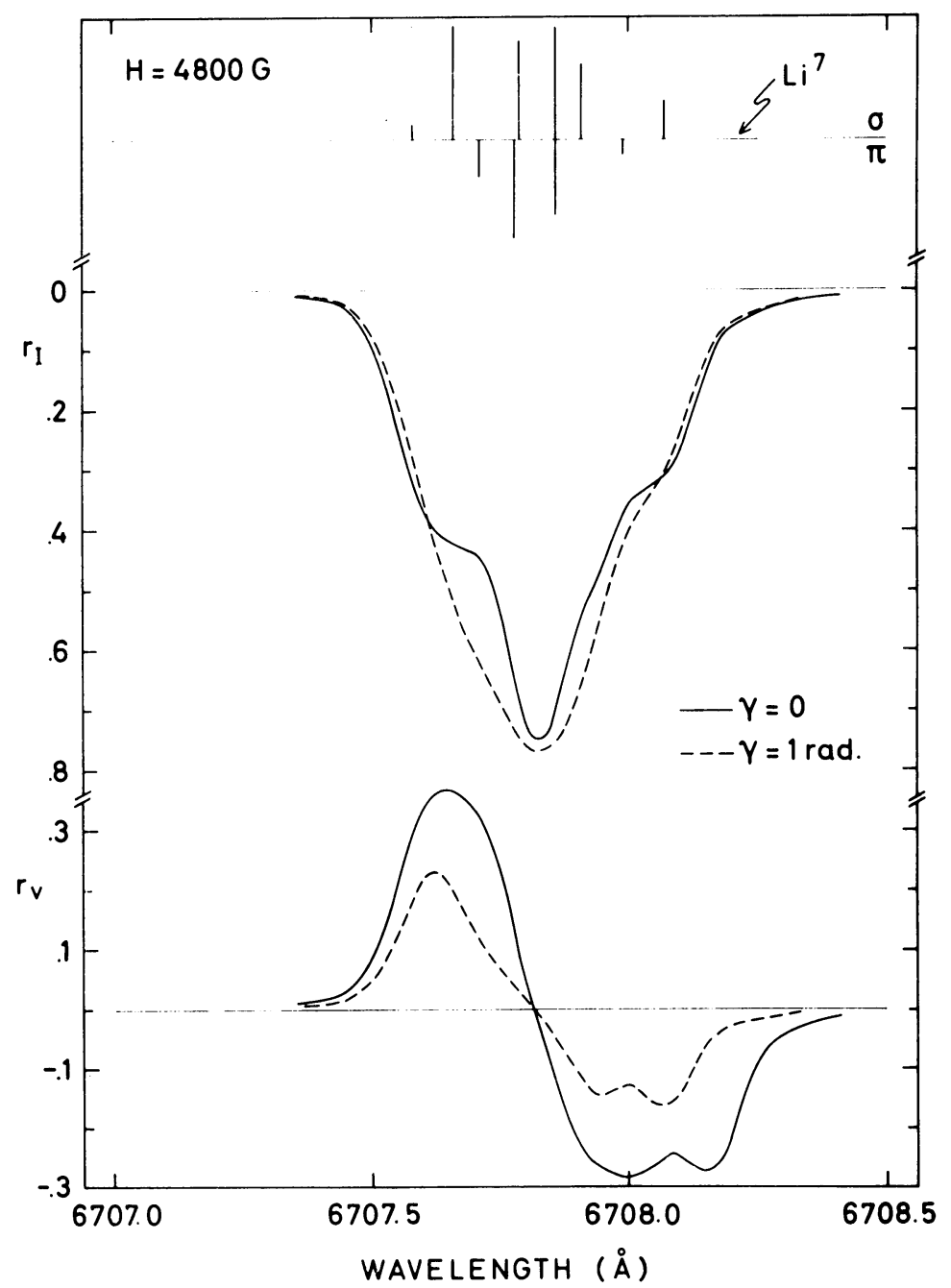

Fig. 3. 
The calculations show that an error in the line profile is introduced if a Zeeman splitting pattern is used instead of the Paschen-Back pattern. The error, $\Delta r_{I}$, in the line depth $r_{I}$ is of the order of 0.01 for a magnetic field strength of $H=2400 \mathrm{G}$, but increases rapidly to $\Delta r_{I} \approx 0.03$ for $H=3300 \mathrm{G}$ and to $\Delta r_{I} \approx 0.10$ for $H=4800 \mathrm{G}$. The errors in $r_{v}$ are comparable in magnitude. Thus, the Paschen-Back splitting must be taken into account for sunspot magnetic fields. For very strong fields $(12000 \mathrm{G})$ we approach a complete Paschen-Back splitting pattern.

Figures 1-4 show considerable changes in the line profile and polarization characteristics as the strength and direction of the magnetic field are altered. Thus, although the Doppler-width of the line is considerable, detailed observations of the Li line may give valuable information about the magnetic field configuration in sunspots.

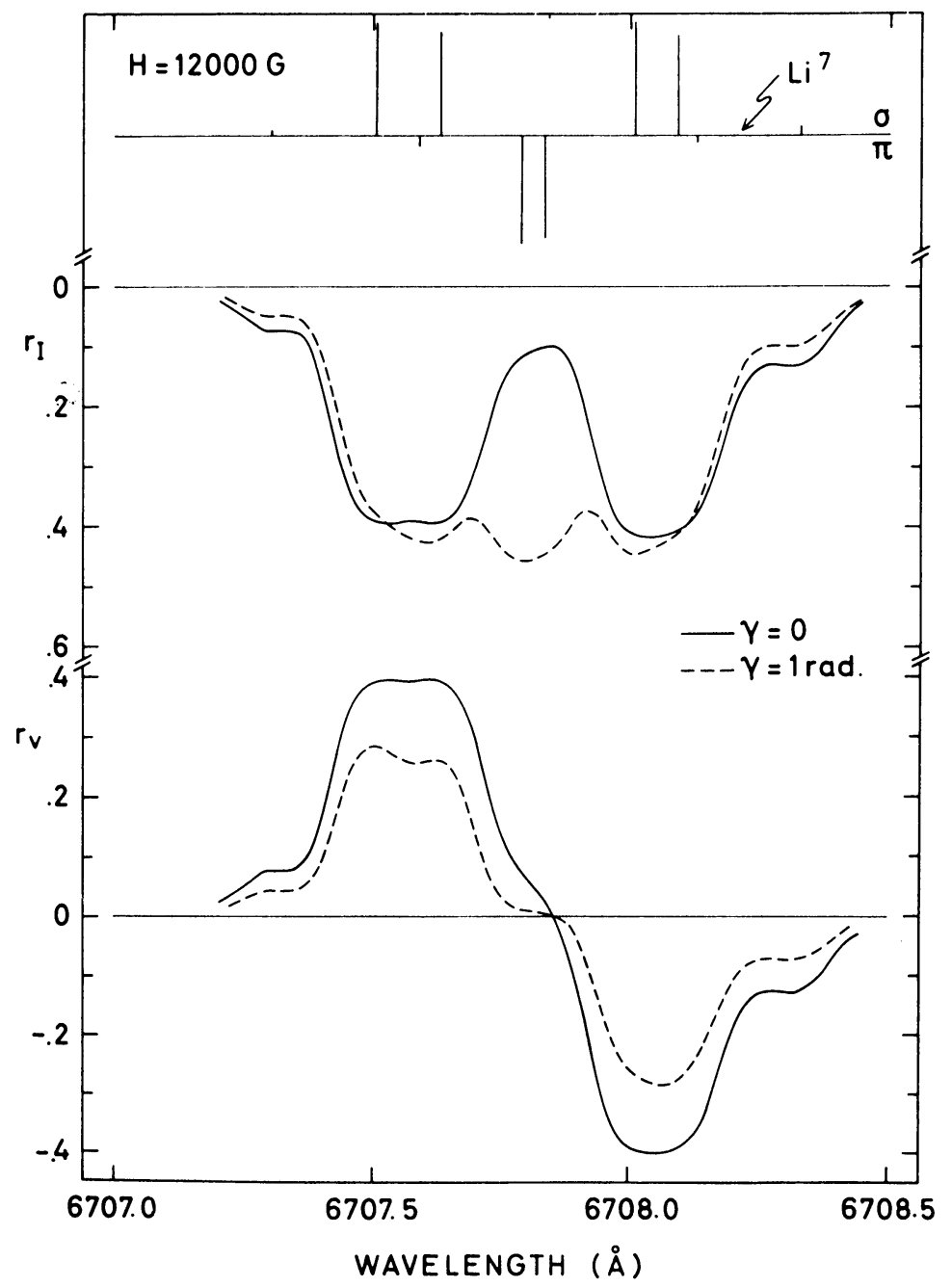

Fig. 4. 
Maltby and Engvold (1970) have recently found that the Li resonance doublet show a faint intensity reversal in sunspot spectra. This probably means that part of the $\mathrm{Li}$ doublet is formed in relatively high layers of the sunspot atmosphere. Accordingly, we must emphasize that the calculations given here should be regarded as a first approximation to the line profile. On the other hand, as the Li doublet very probably is formed in relatively high layers of the spot atmosphere, better line formation theory and observations may give us detailed information about the magnetic field configuration in higher layers of the sunspot atmosphere.

\section{Acknowledgement}

The author wishes to thank O. Engvold for help with the numerical calculations.

\section{References}

Aller, L. H.: 1963, The Atmospheres of the Sun and Stars, Ronald Press, New York, p. 324.

Bethe, H. A. and Salpeter, E. E.: 1957, in Handbuch der Physik (ed. by S. Flügge), Springer-Verlag, Vol. XXXV, p. 291.

Bickel, W. S., Martinson, I., Lundin, L., Buchta, R., Bromander, J., and Bergström, I.: 1969, J. Opt. Soc. Am. 59, 830.

Bode, G.: 1965, Thesis, Kiel.

Darwin, C. G.: 1927, Proc. Roy. Soc. A115, 1.

Darwin, K.: 1928, Proc. Roy. Soc. A118, 264.

Engvold, O., Kjeldseth Moe, O., and Maltby, P.: 1970, Astron. Astrophys. 9, 179.

Greenstein, J. L. and Richardson, R. S.: 1951, Astrophys. J. 113, 536.

Heisenberg, W. and Jordan, P.: 1926, Z. Phys. 37, 270.

Kjeldseth Moe, O.: 1968, Solar Phys. 4, 267.

Maltby, P. and Engvold, O.: 1970, Solar Phys. 14, 129.

Pauling, L. and Goudsmit, S.: 1930, The Structure of Line Spectra, McGraw-Hill, New York, p.74.

Sommerfeld, A.: 1922, Z. Phys. 8, 257.

Traub, W. A.: 1968, Thesis, University of Wisconsin.

Voigt, W.: 1913, Ann. Phys. 41, 403.

Wiehr, E., Stellmacher, G., and Schröter, E. H.: 1968, Astrophys. Letters 1, 181.

Zwaan, C.: 1965, Rech. Astron. Obs. Utrecht 17, (4). 